\title{
Visiones de los agentes implicados en el diseño, difusión y utilización de los materiales digitales educativos en portales educativos españoles
}

\section{Visões dos agentes envolvidos na concepção, divulgação e utilização de materiais educativos digitais nos portais educativos espanhóis}

\section{Visions of the agents involved in the design, dissemination and use of digital educational materials in Spanish educational portals}

\author{
José Peirats Chacón* \\ Raúl Eirín Nemiña** \\ Jesús Rodríguez Rodriguez ${ }^{* *}$
}

\begin{abstract}
RESUMEN
Este artículo recoge resultados de un estudio más amplio dedicado al análisis de las visiones de los agentes y actores implicados en el diseño, difusión y utilización de los materiales digitales educativos presentes en portales educativos españoles que ofertan servicios y recursos educativos. Forma parte, a su vez, de un proyecto de I+D+i, denominado "Escuela Digital", dedicado al análisis de la producción y uso de contenidos didácticos digitales
\end{abstract}

*Universidad de València. València, España. E-mail: jose.peirats@uv.es. https://orcid. org/0000-0002-6580-2712.

** Universidad de Santiago de Compostela. Santiago de Compostela. España. E-mail: raul. eirin@usc.es. https://orcid.org/0000-0002-2990-0905. E-mail: jesus.rodriguez.rodriguez@usc.es. https://orcid.org/0000-0003-4194-2574. 
en los centros escolares ${ }^{1}$. El propósito principal del artículo es presentar los resultados de las visiones del profesorado, el alumnado y las familias sobre los materiales digitales presentes en los portales institucionales de Galicia y Valencia, ofreciendo sugerencias para su mejora. Tras una revisión bibliográfica del ámbito de investigación considerado sobre materiales didácticos digitales y portales institucionales exponemos la metodología de investigación. Luego sintetizamos los resultados del análisis y, finalmente, presentamos la discusión y conclusiones del análisis realizado.

Palabras clave: Materiales didácticos. Tecnología. Profesorado. Familias. Alumnado.

\title{
RESUMO
}

Este artigo recolhe resultados de um estudo mais amplo dedicado à análise das visões dos agentes e atores envolvidos no desenho, difusão e uso dos materiais educacionais digitais presentes nos portais educacionais espanhóis que oferecem serviços e recursos educacionais. Faz parte, por sua vez, de um projeto de I + D + i, denominado "Escola Digital", dedicado à análise da produção e uso de conteúdos didáticos digitais nas escolas. $\mathrm{O}$ objetivo principal do artigo é apresentar os resultados das visões de professores, alunos e familiares sobre os materiais digitais presentes nos portais institucionais da Galiza e Valência, oferecendo sugestões para o seu aperfeiçoamento. Após uma revisão bibliográfica do escopo da pesquisa considerada em materiais didáticos digitais e portais institucionais, expomos a metodologia de pesquisa. Em seguida, sintetizamos os resultados da análise e, finalmente, apresentamos a discussão e as conclusões da análise realizada.

Palabras chave: Materiais de ensino. Tecnologia. Professores. Famílias. Estudantes.

\begin{abstract}
This article collects results of a broader study dedicated to the analysis of the visions of the agents and actors involved in the design, diffusion and use of the digital educational materials present in Spanish educational portals that offer services and educational resources. It is part of an $\mathrm{R}+\mathrm{D}+\mathrm{i}$ project, called "Digital School", dedicated to the analysis of the production and use of digital didactic contents in schools. The main purpose of the article is to
\end{abstract}

1 Este artículo es resultado del proyecto de investigación titulado "La escuela de la sociedad digital: análisis y propuestas para la producción y uso de los contenidos digitales educativos" (EDU2015-64593-R). Financiado por el Programa Estatal de I+D+i Orientada a los Retos de la Sociedad convocado por el Gobierno de España y dirigido por el profesor Manuel Area. 
present the results of the visions of teachers, students and families about the digital materials present in the institutional portals of Galicia and Valencia, offering suggestions for their improvement. After a bibliographic review of the research scope considered on digital didactic materials and institutional portals, we expose the research methodology. Then we synthesized the results of the analysis and, finally, we presented the discussion and conclusions of the analysis carried out.

Keywords: Teaching materials. Technology. Teachers. Families. Students.

\section{Introducción}

A modo de contextualización, conviene indicar que nuestro estudio se sitúa en el marco de la investigación titulada: Escuel@ Digital, la escuela de la sociedad digital: análisis y propuestas para la producción y uso de los contenidos digitales educativos en el que participan tres equipos de investigación de tres Comunidades Autónomas distintas y en el que se "pretende analizar el estado actual de la producción, distribución y utilización pedagógica en las aulas de los contenidos digitales educativos o materiales didácticos online destinados a la Educación Primaria en una muestra de tres comunidades autónomas de España (Canarias, Galicia y Valencia)" (Area, 2015:1).

En el caso concreto de este artículo nos centraremos en realizar una aproximación a la opinión y visión del profesorado, del alumnado y de las familias sobre los materiales didácticos digitales presentes en los portales de las editoriales e institucionales en el marco del proyecto de investigación considerado. Las razones que justifican la elección de los portales y el estudio de los materiales didácticos presentes en los mismos son las siguientes:

- Ofrecen acceso a recursos y materiales didácticos, así como información sobre las características de los mismos.

- Los portales institucionales de las administraciones recogen propuestas de materiales didácticos que, o bien han sido financiados por la propia administración o han sido elaborados por los propios profesores.

- Los materiales digitales incorporan múltiples y variadas formas de representación y muestran diferentes relaciones entre los sujetos participantes y el objeto de estudio.

- Contribuyen a la difusión de los materiales didácticos elaborados.

En cuanto a las argumentaciones que justifican la participación de los agentes seleccionados podríamos destacar las siguientes. En relación con el profesorado, se espera que su implicación en este contexto curricular, suponga un 
compromiso con la utilización de estos materiales. Partimos de la idea de que los materiales digitales, podrían contribuir a considerar a los profesores y profesoras más allá de su condición de técnico encargado de enseñar conocimientos preestablecidos, y favorezcan que pueda constituirse en un profesional con capacidad para filtrar, analizar, criticar, reflexionar y transformar la realidad; un agente de cambio, de quien se espera que observe, explore, experimente, construya sus propios materiales y, en el caso que decida hacer uso de materiales elaborados, lo haga de modo reflexivo y crítico. Sería de esperar que las particularidades de los materiales digitales contribuyan a que el uso de estos recursos se constituya en una oportunidad para el continuo desarrollo de su profesionalidad.

De igual manera y teniendo en cuenta que los materiales digitales conllevan nuevas exigencias y un papel más activo de las familias (Fraga Varela y Duarte Fernández, 2015; Fraga Varela y Alonso Ferreiro, 2016), nos parece muy importante conocer su visión sobre los materiales didácticos que han sido elaborados. Obviamente, en un estudio de las características recogido en este artículo, resulta fundamental conocer la opinión del alumnado como principal destinatario y usuario de los materiales didácticos digitales elaborados.

De un modo concreto, en el caso de nuestro trabajo pretendemos atender a los siguientes objetivos específicos:

- Identificar las representaciones/visiones que los distintos agentes educativos (profesorado, alumnado y familias) tienen sobre el potencial didáctico de los contenidos digitales en la enseñanza primaria.

- Analizar las representaciones que tienen tanto los responsables de gestión de portales/repositorios institucionales como los de empresas productoras de contenidos educativos comerciales sobre el potencial didáctico y el mercado de los contenidos digitales en la enseñanza primaria.

Para atender a estos objetivos hemos desarrollado una metodología cualitativa que, a través de grupos de discusión y entrevistas con los agentes implicados (docentes, alumnado, familias, responsables de empresas y de portales autonómicos en las dos CCAA), intenta dar luz a las representaciones y visiones de los participantes en el estudio.

\section{Revisión de la Literatura}

Con el propósito de contextualizar la investigación realizada, hemos procedido a la revisión de aquellos trabajos y estudios realizados recientemente, y 
que han tenido como perspectiva fundamental de estudio la visión de los agentes señalados en el estudio en relación con los materiales didácticos digitales e igualmente hemos considerado aquellas investigaciones de los últimos años que han estado centradas en el estudio o análisis de los materiales didácticos digitales presentes en portales de editoriales e institucionales.

En relación con los estudios sobre las visiones de los agentes, convendría destacar inicialmente el trabajo de revisión Rodríguez Rodríguez, Bruillard y Horley (2015). Digital Textbooks. What's New?, en el que se recogen algunos trabajos que han estado centrados en el estudio y opinión de los agentes sobre el diseño y la utilización de los materiales didácticos digitales. Igualmente, en el monográfico de la Revista Profesorado, Revista de Currículum y Formación del Profesorado titulado: Ni la defensa ingenua ni la denuncia vacía: contribuciones para el análisis de las relaciones entre los libros de texto impreso y digitales (Coords. Rodríguez Rodríguez, Martínez Bonafé, Braga García, 2016), se analizaron las relaciones entre los libros impresos y digitales. De un modo concreto, en el trabajo de revisión de Rodríguez Rodríguez y Rodríguez Regueira (2016) se recoge una revisión sobre investigaciones relacionadas con el uso de los libros de texto y materiales digitales. Por otra parte, en el trabajo de Gómez Mendoza, Braga García y Rodríguez Rodríguez (2016) Balance y análisis sobre la investigación del texto escolar y los medios digitales. Memorias de la Conferencia Regional para América Latina de la IARTEM. Colombia: Universidad Tecnológica de Pereira varios trabajos del contexto latinoamericano han estado centrados en el análisis de la percepción del profesorado sobre los materiales didácticos digitales.

Igualmente resulta de especial mención por su relación con la temática objeto de estudio en este artículo, el monográfico publicado en la revista Relatec $(16,2)$ De los libros de texto a los materiales didácticos digitales (Coords. Jesús Rodríguez Rodríguez, Manuel Area Moreira, 2017) y en el que se pretendió analizar la transformación de los materiales didácticos escolares en el contexto de la sociedad digital. Algunos de los textos referenciados en el monográfico se refieren a las implicaciones de los materiales didácticos digitales para los diferentes agentes educativos.

Por otra parte, en el trabajo de González, Martín y Vega (2018), se analizó cómo se organiza la producción de materiales didácticos de creación propia de portales institucionales, tomando como referencia la perspectiva de sus principales responsables. Sanabria Mesa, Álvarez Núñez y Peirats Chacón (2017) analizaron si las políticas educativas en la producción y distribución de materiales didácticos digitales prestan especial atención al análisis de la situación de los materiales didácticos en tres Comunidades Autónomas de España. Igualmente resulta de interés el trabajo de Castro Rodríguez, Castro Calvo \& Hernández 
Rivero (2017), en el que las plataformas educativas digitales comerciales españolas destinadas a Educación Primaria.

A modo de síntesis de estos trabajos, conviene destacar que la mayoría de los estudios ponen de relieve la escasa formación del profesorado, tanto en relación con los procesos de elaboración de los materiales didácticos digitales, como en su uso adecuado (Peirats Chacón, Gabaldón Estevan y Marín Suelves, 2018). Igualmente, se evidencia la dependencia que manifiestan los profesores del libro de texto digital en lugar de otro tipo de materiales didácticos digitales (Rodríguez Rodríguez y Martínez Bonafé, 2017). También indican una prevalencia de metodologías tradicionales en relación con el uso de los materiales didácticos digitales (Rodríguez Rodríguez y Rodríguez Regueira, 2016).

Respecto a las familias, los trabajos revisados reflejan claramente el desconocimiento de las potencialidades que ofrecen los materiales digitales y evidencian una escasa formación sobre el tema (Véase por ejemplo los trabajos de Fraga y Alonso, 2016). Igualmente, Sanmartín Alonso y Peirats Chacón (2018), destacan los dispares posicionamientos de los padres ante la incorporación de los nuevos medios. En el caso del alumnado, convendría destacar que en general no se ha favorecido la participación activa del alumnado en la generación de contenidos y que prefieren utilizar los materiales digitales fundamentalmente como recurso para el ocio.

En relación con el sector editorial y los materiales didácticos digitales y el contexto de transición del libro de texto, la tendencia es a apostar por la necesaria convivencia entre los formatos impresos y electrónicos (Véase por ej. San Martín Alonso, 2017). En el trabajo de Castro Rodríguez, Castro Calvo y Hernández Rivero (2017), se pone de relieve que la mayoría de las plataformas de contenidos educativos on-line no han apostado por la verdadera potencialidad del espacio virtual para promover modelos pedagógicos que faciliten la participación, la interactividad/conectividad de la comunidad educativa, así como la integración de metodologías alternativas. Igualmente, la mayoría de los materiales que se ofertan en las plataformas comerciales consisten en libros de texto comerciales enriquecidos, que siguen en general un modelo reproductivo y expositivo.

En torno a la cuestión del análisis de los portales, señalamos algunos resultados de la primera parte del proyecto Escuel@ Digital. En esta investigación se ponen de relieve la oferta de repositorios de materiales educativos y recursos (herramientas informáticas, redes sociales...) para el profesorado con la idea de que incorporen las Tecnologías de la Información y la Comunicación (TIC) en las aulas. Los materiales provienen de elaboraciones de docentes, materiales ejemplares por diversos motivos, premios, etc (Santana, Eirín, Marín, 2017: 45). Por otra parte, se destaca que la producción de materiales educativos digitales se encuentra en un estado un tanto difuso, debido a la cantidad de recursos que 
disponemos a nuestra disposición, y la no existencia de directrices que orienten su uso. Igualmente aparece de relieve la necesaria formación del profesorado para la elaboración de los materiales, teniendo en cuenta que ellos representan una parte importante de la producción de estos materiales y son los encargados de valorar su utilidad en el aula (González, Martín y Vega, 2018). Igualmente, y como subrayan Sanabria Mesa, Álvarez Núñez, y Peirat Chacón (2017) desde las administraciones educativas se están desarrollando políticas educativas que favorezcan la integración de las tecnologías digitales, entre las que destacan el desarrollo de portales educativos con el propósito de difundir propuestas de materiales didácticos digitales. No obstante, se echa en falta el análisis con profundidad de la coherencia, la coordinación y la distribución necesaria entre las distintas acciones y los diferentes contextos y agente educativos para estudiar hasta qué punto estas políticas están impregnando la práctica educativa en las aulas y los centros educativos.

Una de las conclusiones que se pone de relieve es el papel y relevancia que desempeñan los portales "institucionales" en la difusión de los materiales didácticos digitales. En este sentido, conviene destacar que se constituyen en uno de los recursos fundamentales para contribuir a la difusión. Los estudios reflejan la necesidad de conocer la visión y opinión de los agentes sobre los materiales didácticos digitales presentes en los portales. Partiendo de estos resultados y de los objetivos propuestos en la investigación, se ha procedido al desarrollo de la investigación mencionada y cuya metodología y resultados procedemos a comentar a continuación.

\section{Metodología}

La metodología seleccionada, con la pretensión de sistematizar la recogida de datos en el trabajo de campo, ha sido la etnográfica (Goetz y Lecompte, 1988; Nolla, 1997). El objeto de nuestra atención en este texto se ha ceñido a los materiales didácticos digitales (MDD) radicados en una serie de portales educativos españoles, tal y como corresponde al estudio II del proyecto de investigación aludido en páginas anteriores (EDU2015-64593-R), y la muestra a la que hemos dedicado nuestra atención se detalla en una serie de agentes implicados en su diseño, elaboración, distribución y uso. Desarrollamos, seguidamente, con más profundidad estos aspectos. 


\section{Objetivo del Estudio II}

El diseño metodológico del proyecto de investigación adquiere carácter mixto al proponer estudios de diferente naturaleza tanto en objetivos, como en procedimiento o contexto a indagar. En su Estudio II, del que nos ocupamos en estas páginas, nos preguntamos sobre las percepciones que tienen los distintos agentes educativos y sociales implicados sobre los MDD contenidos en los portales educativos. Esta cuestión se concretó como objetivo de trabajo, que señalamos a continuación, y unos procedimientos metodológicos que detallaremos posteriormente:

1. Identificar las representaciones/visiones que los distintos agentes educativos (profesorado, alumnado y familias) tienen sobre el potencial didáctico de los contenidos digitales en la enseñanza primaria.

\section{Instrumentos, muestra y análisis de datos}

Como técnica de recogida de información se acordó utilizar las entrevistas de grupo (Barbour, 2013; Ibáñez, 1986; Krueger, 1991) con los agentes educativos de los niveles de quinto y sexto de primaria en los que se focaliza el proyecto. Para ello se seleccionaron tres grupos, uno de profesorado, otro de alumnado y el tercero con miembros de familias, con tamaño variable de participantes (entre cuatro y ocho), en cada una de las comunidades autónomas participantes (Canarias, Galicia y Valencia). Nueve entrevistas de grupo en total, tal y como se puede observar en la tabla siguiente, para explorar sus visiones o representaciones sobre los MDD. 
TABLA 1: REUNIONES CON ESPECIFICACIÓN DE TIPO DE CENTRO Y NÚMERO DE ENTREVISTADOS

\begin{tabular}{|l|l|}
\hline CANARIAS & Profesorado: docentes (7) de dos colegios públicos y dos concertados. \\
\cline { 2 - 3 } & Alumnado: estudiantes (4) de un colegio público. \\
\cline { 2 - 3 } & Familias: padres y madres (4) de dos centros concertados distintos y uno público. \\
\hline GALICIA & $\begin{array}{l}\text { Profesorado: docentes (5) de cuatro colegios, dos de ellos públicos y dos } \\
\text { concertados. }\end{array}$ \\
\cline { 2 - 2 } & $\begin{array}{l}\text { Alumnado: estudiantes (6) de } 2 \text { colegios, uno de ellos público y el otro } \\
\text { concertado. }\end{array}$ \\
\cline { 2 - 2 } & Familias: padres y madres (5) de cuatro centros públicos. \\
\hline VALENCIA & $\begin{array}{l}\text { Profesorado: docentes (5) de dos colegios, uno de ellos público y el otro } \\
\text { concertado. }\end{array}$ \\
\cline { 2 - 2 } & $\begin{array}{l}\text { Alumnado: estudiantes (5) de dos colegios, uno de ellos público y el otro } \\
\text { concertado. }\end{array}$ \\
\cline { 2 - 2 } & Familias: padres y madres (6) de tres centros concertados distintos y uno público. \\
\hline
\end{tabular}

FUENTE: Los autores.

En el desarrollo de la investigación, una vez acabado el primer estudio, se realizó un I Encuentro de Equipos de Investigación, con objeto de diseñar el Estudio II. Entre los acuerdos adoptados en el evento, se convino que todos los entrevistados fueran usuarios de MDD, ya fueran de carácter comercial como de elaboración propia; se planteó reducir el número de participantes inicial en las entrevistas de grupo a entre cuatro y seis participantes; $y$, con la pretensión de que el proceso de análisis fuera homogéneo entre los tres equipos, se acordó elaborar un protocolo de cuestiones metodológicas, en el que se estableció, entre otras cosas que, para el proceso de reducción de datos obtenidos, el análisis de las entrevistas se realizaría mediante matrices de doble entrada (para categorías y sujetos) (Miles, Huberman y Saldaña, 2014).

En las sesiones de trabajo los investigadores se distribuyeron en tres grupos para abordar las dimensiones a indagar en las entrevistas de grupo. Los documentos elaborados (Guías de entrevistas) se alojaron posteriormente en un espacio virtual (en Google Drive) por el investigador principal durante un tiempo acordado (dos semanas) para anotar sugerencias o modificaciones. Entre las aprobadas, se decidió utilizar un sistema de identificación mediante códigos alfanuméricos para diferenciarlas por equipos, agentes y actores. Más tarde se aprobaron como definitivas en sesión de videoconferencia, concluyendo el proceso de elaboración de categorías o dimensiones. Finalmente, basándose en los guiones, se diseñaron las matrices de reducción de datos a tres niveles: de preguntas, de categorías y comparativas entre agentes. 
A continuación, cada equipo de investigadores contactó en su ámbito de trabajo con los agentes educativos para solicitar su participación en la investigación y acordar fecha y lugar de encuentro para la entrevista. Todas se grabaron en audio y fueron transcritas inmediatamente, eliminando los audios posteriormente tras su posterior revisión y autorización por parte de los entrevistados. El análisis se realizó mediante matrices, según el procedimiento descrito anteriormente.

\section{Resultados}

Presentamos seguidamente una síntesis de los resultados finales de las investigaciones desarrolladas en las comunidades autónomas de Galicia y de Valencia, los correspondientes al tercer contexto territorial (Canarias) se encuentran en otro trabajo independiente de este monográfico. Se organizan en torno a los tres agentes educativos interpelados (profesorado, alumnado y familias) y por ámbitos geográficos; ilustrando el relato con algunas evidencias de entre las recogidas en las entrevistas y seleccionadas de las matrices de análisis, no de las transcripciones, ya que debido a las limitaciones de espacio señaladas por la revista no se han podido presentar en su totalidad.

\section{La visión del profesorado}

Si comenzamos nuestra exposición de resultados por los de Valencia, señalamos su opinión favorable, incluso lo consideran imprescindible en esta sociedad red, hacia el trabajo con dispositivos y recursos digitales en el proceso educativo, "es el mundo en el que se desenvuelven los críos", pero manifiestan que el simple uso no asegura el aprendizaje del alumnado ya que deben tenerse en cuenta múltiples variables. También apuntan a que el trabajo con MDD no implica un cambio radical respecto al trabajo con texto impreso, "yo para lo que más lo utilizo es para buscar información complementaria...", y consideran que aumentar la calidad del aprendizaje implica cambiar el uso del digitalizado para que difiera del impreso y se pueda aprovechar mejor sus múltiples potencialidades.

Esta necesidad de introducir y trabajar con recursos digitales en la escuela es un sentir compartido por los profesores de Galicia. Consideran que la escuela no puede quedarse al margen del desarrollo tecnológico y la posibilidad de utilizar, de forma educativa, los múltiples recursos electrónicos disponibles. Así nos dicen que la tecnología es fundamental para estar actualizados y ser buenos 
profesionales de la docencia, "es importante tener recursos digitales en la escuela porque tenemos que actualizarnos día a día"; "como buenos profesores debemos ponernos al día". Nos recuerdan que los cambios de la escuela del siglo XXI se producen a gran velocidad, de manera que existe mayor distancia entre la escuela actual y la de hace pocos años, por lo que "deberíamos cambiar el chip y poder introducir cosas nuevas y metodología nueva”. Además afirman que, en la sociedad hacia la que caminamos, “(...) la tecnología está ahi y está para quedarse".

Destacan en la comunidad valenciana la enorme versatilidad en el aula, dado que ofrecen muchas posibilidades y, sobre todo, porque favorecen la adaptabilidad en los contenidos lo que facilita la implementación del currículo: "los materiales son más adaptables a la realidad de los niños, que permiten ... que ellos, sobre todo, sean los protagonistas del aprendizaje". Valoran su practicidad para responder a las necesidades del alumnado, aunque recomiendan complementar con otro tipo de materiales manipulativos que aseguren la calidad del aprendizaje. Sin embargo, aunque su presencia en las aulas valencianas es ya habitual, su utilización no es homogénea en las diferentes asignaturas; como ejemplo de las diferencias señalaba un docente: "Yo... en natural y social; en educación física, cuando quiero trabajar alguna técnica concreta que es más fácil que la vean ... que la explique yo, y en la tutoría para trabajar la educación transversal".

En Galicia coinciden en este aspecto al señalar que para establecer una medida de la utilidad y pertinencia de los MDD, recurren a "(...) la "práctica" $y$ "el uso racional" de los recursos que, en último término, serán los que determinan su valor". Abundando en esta idea nos recuerdan que el uso de materiales analógicos y digitales debería apoyarse en las posibilidades y ventajas de cada uno, buscando un cierto equilibrio y procurando que la relación entre los mismos no sea excluyente: "Bueno, yo apuesto por un uso un poco equilibrado de todo. Tampoco se trata de que sea uno frente a otro". "Puedes prescindir [de uno de ellos] pero me parece que sería un error, o sea, los dos son necesarios si son complementarios".

Es interesante señalar que también muestran un cierto espíritu crítico y un buen grado de sentido común al afirmar que:

"Existe un cierto fetichismo tecnológico donde la tecnología se ha convertido un poco en ideología y todo aquello que esté en soporte digital y que sea a través de redes y demás parece que tiene un peso, digamos, superior a otro tipo de recursos, ¿no?”. 
Traen al debate la idea de que si bien los avances tecnológicos pueden parecen positivos, ni todos ni siempre suponen una mejoría. También pueden generar problemas o llevar a transformaciones sociales poco deseables.

"Creo que habría que problematizarla también, entendiendo que a veces supone un avance, supone una mejora y en otras ocasiones puede suponer incluso un problema; o producir transformaciones sociales que, a lo mejor no son lo más deseable".

Volviendo a Valencia, respecto a la formación del profesorado destacan la autoformación, aunque depende de la voluntariedad y señalan la edad como el elemento determinante para el dominio y uso de los MDD y las TIC: "Nosotros somos autodidactas, y autodidactas quiere decir que aprendemos conforme vamos a utilizar. Y para eso influye mucho también la edad". Aunque existen múltiples iniciativas formativas en los propios centros apuntan hacia la enorme cantidad de materiales en la red, lo que corroboraba este docente: "crear y reutilizar. Hay mucha gente muy generosa que guarda los materiales en internet y que puedes adaptarlos. Hay gente muy generosa en el mundo de la enseñanza, de verdad".

Por su parte, en el contexto gallego se comparten las preocupaciones en cuanto a la formación: “(...) algo de formación y algo de búscate la vida como puedas, si quieres pregúntale a tus compañeros”. Sin duda el autoaprendizaje es una estrategia muy usada por los docentes...

"Hombre (...) yo llevo trabajando con ordenadores muchos años. Entonces, (...) no me resultaba novedoso...”; “me gustó siempre y después... alli en $P$., es un pueblo bien pequeñito, y entonces, con mis compañeros y B., que es un máquina, pues bueno, pues aprendes y (...) vas haciendo cosas, $y(\ldots)$ '.

También nos dicen que las editoriales, sí se le insiste, acaban enviando a alguien para que les ofrezca formación y explique el funcionamiento de los materiales, pero sólo de aquellos que le han vendido al colegio: "Pero en cuanto a su material, no en cuanto a hacer una cosa o trabajar con esta aplicación, o con otros, eso no". Finalmente citan a la red de formación de la Consellería, al programa Abalar, en el que han participado los coordinadores TIC; en estas 
jornadas les explican los cambios en la plataforma, las nuevas posibilidades que ofrecía ésta, y las posibilidades para trabajar con las herramientas de autor.

En Valencia manifiestan la necesidad de avanzar en la escuela hacia nuevas metodologías basadas en proyectos y en la coordinación interdisciplinar, además de crear una comisión TIC en las escuelas que facilite la implementación digital: "Se ha creado un departamento TIC, formado por tres personas una de infantil, una de primaria y una de secundaria. Y tratan de actualizar la escuela a ciertas tecnologías... ". No consideran relevante la cuestión económica en un contexto escolar con fuerte implicación familiar, aunque es necesario valorar siempre las distintas posibilidades económicas, veamos un ejemplo: "nosotros elegimos unas tabletas Android de un coste medio, mientras en otros colegios han elegido iPad que son de un coste superior". También señalan como necesario invertir no solo en relación con los dispositivos y los MDD, sino también en infraestructuras y mantenimiento para optimizar el trabajo con TIC.

En el contexto gallego, finalmente, también se pone de manifiesto la necesidad de avanzar en la interdisciplinariedad, puesto que "(...) el funcionamiento ordinario en la mayor parte de los centros no suele desarrollarse interdisciplinariamente". De forma paralela aparece el problema del individualismo fomentado por la política de cada niño con un ordenador y la metodología de enseñanza que enfatiza el trabajo individual...," (...) las aulas de E-Dixgal que he visto generalmente son un trabajo muy individualizado, ¿no? (...) limita a veces la posibilidad de utilizarlo de manera complementaria con más salidas o con otro tipo de organización... ", lo que lleva al docente a interrogarse sobre la socialización que se está imponiendo a los alumnos, cómo indirectamente se fomenta cierta atomización social y cómo una tecnologización de la enseñanza provoca cierta deshumanización que acaba provocando que tengamos que reaprender habilidades sociales que antes se daban por sabidas.

“(...) llegamos a un punto de cierta deshumanización a través de la tecnologización y de algún modo tenemos que reaprender artificialmente habilidades sociales que antes se daban casi naturalmente con tus amigos, con tu familia, con tus vecinos, en un montón de contextos de aprendizaje en el que se aprendía eso, y hoy parece así como que hay que hacer actividades especificas para trabajarlos...".

\section{La visión del alumnado}

En este segundo agente educativo resulta sorprendente, en el contexto valenciano, que la mayoría tiene predilección por el recurso tradicional, con 
argumentos como el presentar un manejo más sencillo y porque no existe dependencia de la conexión, o de la batería; evidencias al respecto son, entre otras:

"Pues yo opto por el sistema más tradicional, los libros porque, por ejemplo, si no funciona o no va la aplicación es más eficaz saber con los libros"; "a lo mejor se puede caer, se rompe, o a lo mejor se bloquea alguna red...".

Sin embargo, alguno también indica argumentos a favor de los MDD, como que ayudan al cuidado del medio ambiente, además de la existencia de aplicaciones útiles para la búsqueda de información, la traducción rápida, y la comodidad en cuanto al transporte y manejo del dispositivo. Mayoritariamente declaran que usan el móvil y la tableta para labores de búsqueda de información, la utilización de los recursos didácticos, la calculadora, o el jugar y escuchar música; como muestra de lo expuesto la siguiente opinión: "Solemos utilizar los móviles, la tele y el ordenador, para ellos trabajar y nosotros para buscar información. Yo lo uso para buscar información, ver vídeos en Youtube..."

A ninguno de los alumnos y alumnas valencianos entrevistados se le permite el uso de los móviles en el centro, solo en actividades puntuales: "Nos dejan llevar el móvil para escuchar música en el autobús de las excursiones, porque suele ser bastante largo el trayecto". Trabajan en distintos espacios, tales como la sala de informática o en las aulas ordinarias y también con diversos dispositivos, como la PDI y las tabletas; pero prefieren utilizar el ordenador porque consideran que es mucho más efectivo: porque fomenta la autonomía del trabajo, ayuda en el proceso de repaso del aprendizaje y en la comunicación profesor-alumno, y adquieren aprendizajes nuevos relacionados con la competencia digital.

También señalan- en Galicia- la preferencia del ordenador frente a la tableta, por la facilidad de escritura que le proporciona un teclado grande. Todos destacan ciertas dificultades, especialmente la sobrecarga de la red y los bloqueos o la dependencia a una red limitada:

"No me cargaba porque había demasiada gente utilizando el internet y no me cargaba, y me tuve que esperar un rato a que la gente se desconectara para yo ya poder entrar"; "tenemos una red en este colegio, pero la verdad es que es bastante, no es muy... no es muy eficaz, entonces enseguida la red se cae", o "la mayoría de las veces falla, y se nos queda bloqueado el ordenador o no funciona el proyector, y eso hace que no hagamos cosas como tendríamos que hacerlas y retrasa un poco corregir deberes y eso". 
En el contexto gallego las herramientas TIC son sobradamente conocidas por los niños y las niñas entrevistadas, tanto dentro del ámbito escolar como familiar. Las utilizan fundamentalmente para el ocio y tiempo libre. Suelen estar compartidos en la unidad familiar, y algunos tienen restricciones en el uso de recursos tecnológicos en sus casas, aunque no todos. "Yo también tengo un ordenador, un PC, una Smart TV, un IPad y un móvil y los utilizo para trabajar y para jugar". "A mí no me dejan utilizar nada por la semana, sólo por el fin de semana". El recurso tecnológico preferido es el ordenador, que destaca fundamentalmente por su facilidad de uso en la escuela para escribir y corregir lo escrito, así como por cuestiones "de peso".

"Bueno (...) a mi me ponen también ejercicios en la libreta y en el libro entonces sería una desventaja porque tendría que copiar todo y hacer todo en la libreta... y tendría que copiar más también”.

"Pues yo con materiales tecnológicos preferiría el ordenador porque me es más cómodo escribir que en el teclado del iPad".

"Pues por ejemplo porque si tienes que llevar lengua, lingua y mate, por ejemplo de deberes... y te puedes llevar una tablet que esté lengua, lingua y mate, pues... yo prefiero la tecnología esa porque.... pesa menos."

Relatan un uso de las tecnologías en la escuela bastante dirigido por el docente, unidireccional, usadas fundamentalmente por el profesorado para la explicación o realización de actividades para toda la clase, y que en buena medida se identifica con un uso tradicional del libro de texto. De hecho, el alumnado define el libro de texto digital como el libro de texto impreso, pero que para la realización de algunas actividades "le puedes dar a un botón". Y también que:

"Bueno es que a nuestros profesores ya les dieron un iPad, o sea que lo tienen todas las clases, de quinto y sexto, en todas las clases lo utilizamos. Se lo dan al profesor y desde el iPad ya pone el libro digital y nos dice los ejercicios que hay que hacer y nos explica desde ahi". 
"Es el libro de texto que tenemos en clase pero digitalmente... O sea, tú coges el ordenador y le metes..., te registras y pones el código del libro y consigues el libro digitalmente que lo tienes en el ordenador".

El acceso a las TIC está limitado en el ámbito escolar, siendo los docentes quienes determinan el uso de los recursos, y la opción de elegir entre unos u otros la tienen fuera de la escuela, cuando tienen que realizar los trabajos. En estos casos es cuando pueden elegir sus fuentes informativas. En estos casos, suelen optar por el uso de Internet: "Hoy, hoy tuve el examen del baile tradicional gallego en Educación Física, y nos mandaron buscar en Youtube la canción para hacerlo mejor, para estudiar".

Por último, en esta parte de la exposición de resultados, apuntamos que la idoneidad de los recursos TIC u otros, la justifican según sus necesidades o intereses, lo que les lleva a no magnificar ni rechazar ninguno de ellos de forma tajante. Piensan que tanto el soporte electrónico y virtual como el impreso o manipulativo no van a desaparecer y seguirán conviviendo.

\section{La visión de las familias}

En general, las familias valencianas entrevistadas valoran que los MDD presentan ventajas como la instantaneidad, adaptabilidad al contexto educativo, comodidad y variedad: "Están muy adaptados a nuestro colegio y a nuestro contexto"; "son mucho más variados, (...) están totalmente seleccionados por su maestra". No obstante, existen posiciones enfrentadas; así mientras unos prefieren los tradicionales por su carácter tangible, al tiempo que consideran a los artefactos electrónicos como distractores: "pueden acceder a contenidos que se encuentran fuera del propósito que les ocupa", aunque reconocen que se pueda limitar el acceso a páginas o aplicaciones; y otros también destacan que, a pesar de su carácter motivador, pierden esta característica por su excesiva utilización. La parte contraria señala que el uso de los MDD enriquece el aprendizaje, y la tableta es una herramienta útil que les permite realizar sus actividades con MDD, además de poder utilizarla con finalidad creativa e innovadora, tal y como señalaba una madre: "Mi hija está más motivada porque (...) hay mucho material que es de creación propia; un libro de texto no permite tanta creación propia”.

Las valoraciones que dan las familias de Galicia, también muestran una dualidad entre aquellos favorables al uso de los MDD, argumentando que los nuevos conocimientos que se realizan a través de la tecnología requieren de habilidades, estrategias y capacidades que el libro tradicional no proporciona..., "no cabe duda de que vamos hacia un mundo diferente en el que se están 
produciendo aprendizajes que antes se desconocian y eso solamente se puede hacer a través de la tecnología. Precisan habilidades que el libro tradicional no enseña", y los que muestran una opinión negativa hacia su uso, apoyándose en que no deja de ser un libro de texto digitalizado, con sus defectos, que no cambia la metodología docente.

"yo estoy de acuerdo con que un libro digital es una copia de un libro, pero además es una copia mala porque pierde ciertos aspectos como la rapidez, la forma global de ver todo el conjunto..., pienso que el libro digital, tal y como está ahora es una copia mala. Pierde ciertas propiedades que tiene el papel y no aporta, la mayoría de las veces no aporta nada nuevo”. "(...) es una herramienta que no está bien rematada”.

También aparece el argumento económico, al comparar el material digital y los libros de texto tradicionales, “(...) pienso que hay motivos económicos, sí, porque evitas que los alumnos compren libros. Me da la sensación de que es un motivo de peso, porque sabemos lo que cuestan, y si un profesor se decanta por este tipo de material, pues es gratuito".

La implicación de las familias en el proceso de enseñanza aprendizaje suele ser de acompañamiento y seguimiento de las tareas de sus hijos, si bien comentan que les resulta más complicado hacerlo sin los libros de texto o apuntes puesto que..., "al no tener un soporte donde pueda ver lo que están dando, al no tener unos apuntes escritos en la libreta, no sé si los ha hecho bien o mal (...), para mí es más complicado, no tengo una referencia de un libro donde pueda seguir al profesor"; "cuando hacen un examen en el ordenador no sabes si lo hacen bien a la primera, a la séptima o si simplemente jugaron a la lotería”.

La mayoría de las familias disponen de diversos equipamientos (ordenador, tableta, móviles...), si bien se decantan por el ordenador para hacer las tareas escolares, dejando los demás aparatos para cuestiones lúdicas, “(...) no contemplo la tablet como un tema de trabajo porque lo veo como algo más lúdico o para consultar internet, así una consulta rápida".

En Valencia, la mayoría de las entrevistadas admite no participar demasiado en el proceso de enseñanza y aprendizaje con MDD, y únicamente realizan tareas de búsqueda de información, además de supervisar y controlar de manera puntual las actividades escolares de sus hijos. Como aspecto más relevante apuntan hacia la facilidad de comunicación y el intercambio de información con el profesorado y el equipo directivo del centro, aunque en este aspecto también encontramos alguna opinión divergente: "Es mejor el cara a cara". 
Todas las familias que integran el grupo consultado cuentan con dispositivos móviles (tabletas y teléfonos) y ordenadores en sus hogares. En sus centros de referencia han tenido que adquirir las tabletas que utilizan sus hijos, a lo que una de las entrevistadas señalaba que "deberían de plantear algún tipo de ayuda para las tablet”. Sobre el uso de estos dispositivos, estiman como más económico el utilizar MDD elaborados por el propio profesorado de la escuela, a diferencia de los libros de texto digitalizados, como ejemplo esta opinión:

"La maestra pensó que ya teníamos bastante gasto con comprar la tablet, como para hacernos tener el gasto extra de comprar los libros digitales que, además comprabas la licencia, no sé si eran 12 euros por libro, pero al año ya ni te valían".

Finalmente, todas las familias coinciden en que, comparando el coste de la inversión en la tableta con la adquisición de lotes de libros tradicionales de años anteriores, el desembolso no ha sido mayor, por lo que estiman que la operación le sale a cuenta.

\section{Conclusiones}

En este artículo hemos pretendido dar a conocer la opinión de los agentes educativos, es decir del profesorado, el alumnado y las familias de dos contextos territoriales españoles, Galicia y Valencia, en el marco de un estudio específico llevado a cabo en una investigación $\mathrm{I}+\mathrm{D}+\mathrm{i}$ financiada por el programa nacional. Aunque distantes entre sí y con lenguas vehiculares propias diferenciadas, hay muchos puntos que coinciden sobre el diseño, difusión y utilización el uso de los materiales didácticos digitales en las aulas y los hogares, aunque también hemos resaltados ciertas particularidades que enriquecen los hallazgos, veamos a continuación qué se puede señalar para concluir.

Las visiones de los profesores destacan por una posición favorable al uso de los MDD en la escuela, apoyado en la necesidad de preparar al alumnado para el entorno tecnológico en que les ha correspondido vivir y la omnipresencia de las TIC en la sociedad actual. Valoran la practicidad, versatilidad, motivación y cercanía de los materiales a las realidades del alumnado, si bien recomiendan que se complementen con otro tipo de materiales textuales y manipulativos 
que aseguren el aprendizaje, buscando un cierto equilibrio y procurando que la relación entre los mismos no sea excluyente. También muestran su preocupación hacia la necesidad de una mayor interdisciplinariedad en la escuela que pueda sacar todo el potencial transversal que tiene el uso de las TIC.

Los docentes muestran sentido común y espíritu crítico al no dejarse envolver por el fetichismo tecnológico. Reconocen que, si bien los avances tecnológicos permiten mejoras en las vidas de las personas -en nuestro caso las situaciones de enseñanza y aprendizaje que concurren en la escuela-, también pueden generar problemas y transformaciones sociales poco deseables. Es por tanto necesario actuar reflexivamente y valorar con cautela las decisiones que se toman. En este sentido muestran su preocupación por un cierto individualismo que fomenta el trabajo con estos materiales y la necesidad de aprendizaje de habilidades, especialmente sociales, que hasta hace bien poco se daba en los contextos próximos al alumnado, como el patio del colegio, el barrio, la familia, amigos, etc. Respecto a la formación, señalan como agentes implicados en la misma a la administración y a las empresas editoriales, si bien aluden fundamentalmente a la autoformación y la colaboración entre compañeros como la vía de mejora de sus competencias.

Los estudiantes nos indican con naturalidad que trabajan con recursos digitales y también con materiales clásicos. Algunos prefieren aquellos más tradicionales -libros de texto- principalmente para evitar los problemas de bloqueo, falta de conexión, descarga de batería, etc, y la inmediatez de uso que representa el libro de texto. De sus opiniones, subyace la idea de que no hay diferencia sustantiva entre materiales al ser los MDD, generalmente, una copia en pdf del libro de texto tradicional. Todos ellos conocen y manejan diversos dispositivos (móvil, tableta, tv con conexión, calculadoras,...) tanto a nivel escolar como en el hogar, si bien en el colegio tienen ciertas restricciones (especialmente con el móvil), y mayoritariamente se decantan por preferir el ordenador frente a los demás dispositivos, argumentando que fomenta la autonomía del trabajo, ayuda en el proceso de repaso del aprendizaje, en la comunicación profesorado-alumnado y estimula aprendizajes nuevos relacionados con la competencia digital. También señalan la mayor comodidad que supone a la hora de escribir.

Reconocen las posibilidades de los recursos TIC a la hora velar por el medio ambiente (por ejemplo, el no gastar papel), búsqueda inmediata de información, peso y transporte más cómodo que la mochila de libros o incluso la traducción rápida, pero intuimos que el modelo docente que subyace es clásico, apoyado en las decisiones del profesorado y el trabajo tradicional con el libro de texto, por lo que no se explora el potencial de estos MDD y la tarea habitual en el aula se ajusta en un hacer lo mismo de siempre, pero con otros dispositivos. En este sentido, como ya hemos destacado en el apartado de resultados, el alumnado 
nos habla de un uso unidireccional de las tecnologías dirigido por el profesor, haciendo una traslación de las rutinas y tareas realizadas con el libro de texto al material electrónico. Las tareas docentes habituales, las explicaciones de los temas, la propuesta de actividades a realizar, la corrección de las mismas, se identifican en buena medida con lo realizado al usar el libro de texto tradicional. Es por ello que intuimos que no muestran una preferencia muy marcada hacia el soporte electrónico o impreso, y además piensan que ninguno de los dos va a desaparecer, sino que seguirán conviviendo.

En general las opiniones de las familias son favorables al uso de las TIC y los MDD en la escuela. Entienden que presentan la ventaja de un acceso inmediato a la información, la comodidad de acceso y la variedad de recursos educativos que pueden utilizarse. También argumentan que los niños y niñas necesitan de nuevos conocimientos que se realizan a través de la tecnología, que requieren de habilidades, estrategias y capacidades que el libro tradicional no proporciona, pero tampoco un material digital si es un pdf del libro de texto.

No obstante, también aparecen opiniones contrarias al uso de los MDD y TIC. Muestran la preferencia por los materiales clásicos (libros de texto), por ser un objeto conocido sobre el que tienen control, y además por costumbre y apoyados en la creencia de que los dispositivos electrónicos actúan como distractores de la atención necesaria para el estudio. También señalan que el supuesto carácter motivador de los aparatos electrónicos, se pierde en cuanto el alumnado lo usa cotidianamente.

El nivel de implicación de las familias en el trabajo docente suele circunscribirse al acompañamiento y seguimiento de las tareas de sus hijos, así como al contacto puntual con los docentes. Para la ayuda al estudio y acompañamiento señalan que prefieren hacerlo con los materiales tradicionales, que utilizan como guía del trabajo de sus hijos; consideran que este seguimiento de las tareas escolares es más complejo y difuso cuando usan MDD. En cambio, destacan las ventajas de los elementos electrónicos para facilitar la comunicación y el intercambio de información con los docentes, si bien otras opiniones prefieren un encuentro personal para tratar temas de mayor trascendencia y dejar los dispositivos electrónicos para avisos y comunicaciones.

En cuanto al tema económico, el pago de los materiales didácticos asociado a la gratuidad de la enseñanza ha constituido siempre una reclamación histórica de las familias. En nuestro estudio encontramos dos modelos diferentes, correspondientes con los territorios en que se realiza el estudio. En Valencia, las familias seleccionadas en el estudio soportan el gasto de compra de las tabletas, tanto en la escuela pública como en la concertada; sin embargo, sobre la compra de los materiales a utilizar en los dispositivos (libros de texto digitales, materiales y recursos complementarios...) tienen la posibilidad de acogerse al programa 
autonómico gratuito de banco de libros (XarxaLlibres) o son elaborados por profesores o de uso libre en la red, por lo que se ahorran la compra de los libros de texto. En Galicia, aquellos centros y cursos adheridos a los programas de introducción de material digital (E-Dixgal), reciben los aparatos y materiales de trabajo de forma gratuita, mientras que los centros y cursos fuera del programa, las familias sufragan estos gastos.

\section{REFERENCIAS}

Area, M. (2015). Resumen Memoria Técnica del Proyecto Escuel@ Digital (EDU201564593-R). La escuela de la sociedad digital: análisis y propuestas para la producción y uso de los contenidos digitales educativos, financiado por el Plan Estatal de I+D+i. I.P. Manuel Area (Documento oficial, difusión restringida).

Barbour, R. (2013). Los grupos de discusión en Investigación Cualitativa. Madrid: Ediciones Morata.

Castro Rodríguez, Ma . M., Castro Calvo, A., Hernández Rivero, V. (2017). Análisis de plataformas educativas digitales en España. Los casos de Canarias, Galicia y Valencia. Relatec, 16, 2, 49-62.

Fraga Varela, F. y Alonso Ferreiro, A. (2016). Presencia del libro de texto digital en Galicia. Una mirada estadísticogeográfica del proyecto e-dixgal. Profesorado: Revista de curriculum y formación del profesorado, 20, 1, 91-112.

Fraga Varela, F. y Duarte Fernández, A. (2015). La perspectiva de las familias de un centro educativo digital con modelo 1 a 1: implantación del proyexo E-DIXGAL como desarrollo del Plan Escuela 2.0 y el Proyecto Abalar en Galicia. Innovación Educativa, $25,309-325$.

Goetz, J. P. y Lecompte, M. D. (1988). Etnografia y diseño cualitativo en investigación educativa. Madrid: Morata.

Gómez Mendoza, M. A., Braga García, T. y Rodríguez Rodríguez, J. (2016). Balance y análisis sobre la investigación del texto escolar y los medios digitales. Memorias de la Conferencia Regional para América Latina de la IARTEM. Colombia: Universidad Tecnológica de Pereira.

González Ruiz, C. J., Martín Gómez, S. y Vega Navarro, A. (2018). Portales educativos: la producción de materiales didácticos digitales. @tic. Revista d'Innovació Educativa, 20, 89-97.

Ibáñez, J. (1986). Más allá de la sociología. El grupo de discusión: teoría y crítica. Madrid, Siglo XXI Editores. 
Krueger, R. A. (1991). El grupo de discusión. Guía práctica para la investigación aplicada. Madrid, Pirámide.

Miles, M. B., Huberman, A. M. y Saldaña, J. (2014). Qualitative Data Analysis: A Methods Sourcebook. Los Angeles: Sage.

Nolla Cao, N. (1997). Etnografía: una alternativa más en la investigación pedagógica. Educ. Med. Super., 11, 2, 107-115.

Peirats Chacón, J., Gabaldón Estevan, D. y Marín Suelves, D. (2018). @tic. Revista d'Innovació Educativa, 20, 54-62.

Rodríguez Rodríguez, J. y Area Moreira, M. (2017) (Coords.). De los libros de texto a los materiales didácticos digitales. (Monográfico). Relatec, 16 (2).

Rodríguez Rodríguez, J., Bruillard, E. y Horley, M. (Eds) (2015). Digital Textbooks. What's New? Santiago de Compostela: IARTEM, Servizo de Publicacións USC.

Rodríguez Rodríguez, J. y Martínez Bonafé, J. (2017). Libros de Texto y control del curriculum en el contexto de la sociedad digital. Cad. Cedes, 36,100, 319-336.

Rodríguez Rodríguez, J., Martínez Bonafé, J., Braga García, T. (2016) (Coords.). Entre libros de texto impresos y libros de texto digitales: debate y desafío. Profesorado. Revista de Currículum y Formación del Profesorado. (Monográfico), 20, 1.

Santana Bonilla, P. J., Eirín Nemiña, R., Marín Suelves, D. (2017). Análisis y evaluación de portales institucionales en España. Los casos de Canarias, Galicia y Valencia. Relatec, 16, 2, 29-48.

Rodríguez Rodríguez, J. y Rodríguez Regueira, N. (2016). Revisión de la investigación publicada sobre el libro digital en Revistas, publicaciones y congresos internacionales de referencia. Profesorado. Revista de Currículum y Formación del Profesorado, 20 (1), 9-31.

Sanmartín Alonso, A., y Peirats Chacón, J. (2018). Controversias en la transición del libro de texto en papel y electrónico a los contenidos digitales. RED: Revista de Educación a Distancia, 56, 1-17.

Sanabria Mesa, A. L., Álvarez Núñez, Q. y Peirats Chacón, J. (2017). Las políticas educativas en la producción y distribución de materiales didácticos digitales. Relatec, $16(2), 63-77$.

San Martín Alonso, A. (2017). Entrevista a Dolors Pedrós Comany, Presidenta de la Associació d'Editors del Pais Valencià: “el papel y los formatos electrónicos pueden convivir perfectamente, ese debería ser el futuro". Comunicación y pedagogia: Nuevas tecnologías y recursos didácticos, 299-300, 7-10.

Texto recibido el 06/08/2019.

Texto aprobado el 01/09/2019. 